\title{
Consecuencias del COVID-19 en la desigualdad presupuestaria de los equipos de Primera División de LaLiga en España Consequences of the COVID-19 on the budgetary inequality of the First Division teams of LaLiga in Spain
}

David Almorza Gomar, Manuel Huertos César Universidad de Cádiz (España)

\begin{abstract}
Resumen. En este trabajo analizamos los presupuestos de los equipos de fútbol de la Primera División de LaLiga en España durante las temporadas 2019-2020 y 2020-2021, es decir, antes y después del COVID-19. Se realiza una comparación mediante el Índice de Gini y la Curva de Lorenz, observándose la existencia de una gran desigualdad que ha disminuido un poco debido a las repercusiones económicas que ha tenido la pandemia (disminución de los ingresos de taquilla, gastos derivados de la prevención sanitaria, etc.). Esta disminución, haciendo un paralelismo en comparación con la desigualdad económica que existe entre países, y que es medida y publicada por el Banco Munidal a través del Índice de Gini, hace que, de un total de ciento cincuenta y seis países, LaLiga haya pasado de la tercera a la séptima posición. Una gran desigualdad, en cualquier caso, que tiene repercusiones deportivas tanto en el panorama nacional como en el internacional.
\end{abstract}

Palabras clave: fútbol, presupuesto, índice de Gini, curva de Lorenz, desigualdad, COVID-19.

\begin{abstract}
In this paper we analyze the budgets of LaLiga First Division football teams in Spain during the 2019-2020 and 2020-2021 season, it is said, before and after COVID-19. A comparison is made using the Gini Index and the Lorenz Curve, and it is observed that exists a great inequality that has decreased due to the economic repercussions of the pandemic (decrease in tickets office income, expenses derived from health prevention, etc.). This decrease, compared to the economic inequality that exist among countries, and that is measured and published by World Bank through the Gini Index, means that of a total of one hundred and fifty-six countries, LaLiga has gone from third to seventh position. A great inequality in any case, which has sporting repercussions both on the national and international scene.
\end{abstract}

Palabras clave: soccer, budget, Gini index, Lorenz curve, inequality, COVID-19.

\section{Introducción}

En España el fútbol profesional genera un impacto importante en la actividad económica. Según se recoge en el informe de PCW (2018), equivale al 1,37\% del Producto Interior Bruto. En euros, generó una actividad económica de 15.688 millones de euros durante la temporada 2016-2017. En esa misma temporada se contabiliza a cerca de 185.000 personas empleadas en esta industria (PCW, 2018). Es una cantidad que ha ido en aumento en pocos años. A modo de referencia, en la temporada 2005-2006 la industria española del fútbol profesional sería responsable de casi 66.000 puestos de trabajo (García del Barrio \& Pujol, 2008), lo que ha supuesto un incremento considerable en pocos años.

Los ingresos que reciben los clubes de fútbol de Primera División cada temporada tienen dos fuentes principales: los derechos de televisión y la comercialización

Fecha recepción: 02-04-21. Fecha de aceptación: 22-09-21

David Almorza Gomar

david.almorza@uca.es de productos. En la temporada 2011-2012 estos dos conceptos suponían casi el 70\% de los ingresos (Gay de Liébana, 2012). Unos años después, en la temporada 2016-2017 y solo para los veinte equipos del mundo que más dinero generan, esos dos conceptos suponían casi el 85\% de los ingresos (Boor, Hanson \& Ross, 2018).

Si nos ceñimos a los presupuestos de los equipos de fútbol de la Primera División de LaLiga en España, y como posteriormente se demostrará, se constata que ofrecen una gran desigualdad. En la temporada 2019. 2020 los presupuestos oscilaban entre los 1047 millones de euros del F.C. Barcelona y los 32 millones de euros del Real Valladolid (Arés, 2020). Casi treinta y tres veces más. Un nivel de desigualdad prácticamente insalvable.

En las últimas veinte temporadas, excluyendo la que acaba de concluir, que analizaremos por separado como caso especial tras el efecto COVID-19, solo en tres ocasiones ha logrado ser campeón de la Primera División en LaLiga un equipo diferente del Real Madrid y del F.C. Barcelona (AS, 2020), precisamente los dos equipos con mayor presupuesto. Esta situación hace que el 
resto de los equipos de Primera División, más que jugar para ganar la liga, luchen por alcanzar una plaza para jugar la Champions de las que no ocupen Real Madrid y F.C. Barcelona (Herrera, 2016).

La idea de una competición en igualdad de oportunidades hace tiempo que dejó de existir, pero las diferencias económicas se están volviendo abrumadoras. La aparición del COVID-19 ha afectado de manera principal a los equipos con presupuestos más altos Nos preguntamos si hasta el punto de haber disminuido la desigualdad presupuestaria.

El COVID-19 ha intervenido en casi todas las actividades humanas, y también en las deportivas. Aunque se trata de una pandemia reciente, se han realizado estudios sobre sus efectos en el deporte. Desde la faceta de la educación física, Baena-Morales, López-Morales \& García-Taibo (2021) y Hortigüela-Alcalá, HernandoGarijo \& Pérez-Pueyo (2021), o en el contexto de la actividad física de los estudiantes universitarios (Alarcón \& Hall-López, 2021) e incluso en la práctica deportiva de algunos deportes en concreto como la carrera a pie o el ciclismo (Segui-Urbaneja, Juliao, Nogueira, Dorado \& Farias-Torbidoni, 2021).

En Primera División, desde la Real Federación Española de Fútbol (RFEF), la comisión de seguimiento del COVID-19 tomó la decisión de suspender la competición a partir del día 13 de marzo de 2020 (RFEF, 2020). La competición se retomó finalmente el día 11 de junio de 2020 y finalizó el día 19 de julio de 2020 (Fuentes, 2020).

Con la prohibición de la asistencia de público a los estadios, los ingresos por taquilla disminuyeron. El Real Madrid, por ejemplo, con un ingreso medio por partido de 5,3 millones de euros recauda, solo en entradas, cerca de 132 millones de euros por temporada. El F.C. Barcelona, con un ingreso de 4,6 millones de euros en taquilla por partido, recauda aproximadamente 130 millones de euros por temporada. Lo que hace un total de 262 millones de euros que solo estos clubes dejaron de ingresar con motivo del COVID-19. En total, añadiendo los ingresos por marchandising, publicidad y otras partidas que, de forma indirecta, suponían ingresos en los clubes, se estima unas pérdidas para los veinte equipos de Primera División de unos 680 millones de euros (Coll, 2020), que afectará más a aquellos equipos con mayor presupuesto.

Ante esta situación los equipos no tuvieron más remedio que reconsiderar sus presupuestos, que en la temporada 2020-2021 oscilaban entre los 733 millones de euros del F.C. Barcelona y los 43 millones de euros del
Cádiz C.F., una diferencia mucho menor que la temporada anterior.

El objetivo de este trabajo es analizar la desigualdad entre los presupuestos de los equipos de la Primera División y comparar cómo ha variado como consecuencia del COVID-19.

El análisis de la desigualdad presupuestaria se abordará desde el punto de vista gráfico mediante la Curva de Lorenz, y de forma analítica mediante el Índice de Gini.

La Curva de Lorenz fue introducida en el año 1905 por el economista americano Max Otto Lorenz (Upon \& Cook, 2014). El cociente entre el área que determina la Curva de Lorenz y la línea de equidistribución (bisectriz del primer cuadrante), y el área total por debajo de esa línea, se conoce como Índice de Gini o Coeficiente de Gini (Upon \& Cook, 2014). Este índice se debe a Corrado Gini que lo presentó por primera vez en el año 1912 (Ceriani \& Verme, 2014). Los valores del Índice de Gini están comprendidos entre 0, que sería el caso de una equidistribución o máxima igualdad de presupuestos, y 1, que indicaría la situación de máxima desigualdad.

Aunque nacido para medir la desigualdad económica, el Índice de Gini ha sido adaptado y utilizado en el fútbol para medir la desigualdad de puntos que alcanzan los equipos en las competiciones. Burillo, Pérez-González \& Salinero (2012) relacionan el porcentaje de puntos de una liga con el porcentaje de equipos necesarios para llegar a ese porcentaje de puntuación. Consideran cinco ratios de concentración: porcentaje de puntos del primer clasificado sobre el total de puntos; porcentaje de puntos de los dos primeros clasificados sobre el total de puntos y así hasta el quinto nivel. Concluyen así que a un mayor valor del Índice de Gini obtenido con estos datos, peor es el balance competitivo de esa liga.

También puede calcularse el Índice de Gini sin considerar las ratios de concentración, y tratar con tantos niveles como equipos participen en la competición, como hacen Pérez-González, Pérez-Espés, Cazurro \& Gálvez (2016) o también Mon \& Rodríguez (2016).

En este trabajo vamos a presentar una aplicación del índice de Gini al fútbol, pero a partir del análisis de la desigualdad económica en los presupuestos de los equipos que participan en la Primera División, es decir, en el sentido original de este índice, como ya anticipamos en Huertos y Almorza (2021). Además, se compara el efecto que ha tenido el COVID-19 en los presupuestos de estos equipos. 


\section{Análisis de la desigualdad presupuestaria}

Se toman como referencia los presupuestos de los equipos de la Primera División de LaLIga en la temporada 2019-2020. Los datos se recogen de Arés (2020) y se incluyen en la Tabla 1.

\begin{tabular}{|c|c|c|c|}
\hline $\begin{array}{c}\text { Equipo } \\
\text { (temporada 19-20) }\end{array}$ & $\begin{array}{c}\text { Presupuesto } \\
\text { (millones de euros) }\end{array}$ & $\begin{array}{c}\text { Equipo } \\
\text { (temporada 19-20) }\end{array}$ & $\begin{array}{c}\text { Presupuesto } \\
\text { (millones de euros) }\end{array}$ \\
\hline Real Valladolid & 32 & R.C.D. Espanyol & 88 \\
\hline R.C.D. Mallorca & 33 & Real Sociedad & 112 \\
\hline C.D. Leganés & 52 & Athletic Club & 132 \\
\hline S.D. Eibar & 54 & Villarreal C.F. & 135 \\
\hline C.A. Osasuna & 56 & Real Betis & 162 \\
\hline Granada C.F. & 60 & Valencia C.F. & 183 \\
\hline Deportivo Alavés & 65 & Sevilla F.C. & 185 \\
\hline Levante U.D. & 80 & Atlético de Madrid & 500 \\
\hline Getafe C.F. & 82 & Real Madrid & 822 \\
\hline R.C. Celta & 83 & F.C. Barcelona & 1047 \\
\hline
\end{tabular}

Aplicando el mismo procedimiento a los presupuestos de los equipos de Primera División en la temporada 2020-2021, es decir, unos presupuestos que han sido ajustados por las consecuencias económicas que se han derivado de la pandemia del COVID-19, y que se recogen en la Tabla 2, tomados de Arés (2020).

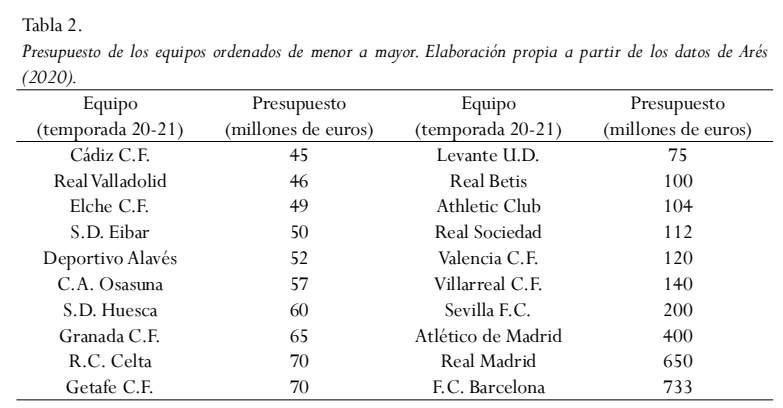

La gráfica de la figura 1 ha sido elaborada con Excell y representa la Curva de Lorenz correspondiente a la desigualdad presupuestaria de las temporadas 2019-2020 y 2020-2021. En ella se aprecia cómo la desigualdad presupuestaria ha disminuido un poco de un año para otro, porque se acerca más a la línea de equidistribución.

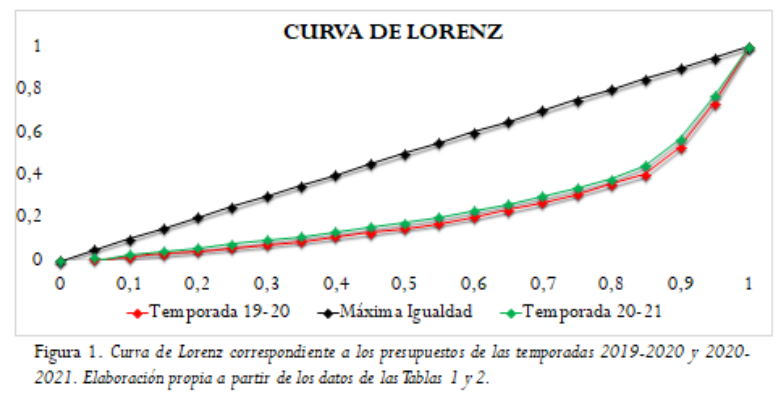

Para calcular el valor correspondiente al Índice de Gini se empleará la siguiente expresión (Montero, 2007):

$$
I G=\frac{\sum_{i=1}^{N-1}\left(p_{i}-q_{i}\right)}{\sum_{i=1}^{N-1} p_{i}}
$$

donde $N$ es el número de equipos que han participado en el estudio; $p_{i}$ es la proporción acumulada de la variable presupuesto y $q_{i}$ es la proporción acumulada de la variable equipo. Tras realizar los cálculos, se llega a un valor del Índice de Gini de 0,588, que se puede considerar como muy elevado.

En este caso, el valor del Índice de Gini que se obtiene a partir de los datos de la Tabla 2, es algo menor y llega a 0,540 , pero sigue siendo un valor muy elevado. Para tener una representación más visual de qué significan estos valores $(0,588$ y 0,540$)$, podemos asociar esta desigualdad presupuestaria de los equipos con la desigualdad económica que existe dentro de los diferentes países.

No es algo nuevo, ya entre las décadas de 1960 y 1970, se acometió el estudio de la desigualdad entre países mediante el Índice de Gini (Coll \& Guijarro, 1998). Este estudio de desigualdad continúa realizándose hoy día y el Banco Mundial publica el ranking de ciento cincuenta y seis países a partir de la desigualdad en función de la distribución del ingreso y medido por el Índice de Gini (IndexMundi, 2020). En la Tabla 3 aparecen los países con mayor desigualdad económica medida por el Índice de Gini.

En la Tabla 3 se aprecia cómo la desigualdad presupuestaria en los equipos de LaLiga durante la temporada 2019-2020 es comparable a la desigualdad presupuestaria entra Namibia y Surinam. En la Tabla 4 se ordena, con el nombre de LaLiga, la situación comparativa de la desigualdad entre los equipos de Primera División esa temporada.

En la Tabla 5 se observa cuál sería la situación de LaLiga en cuanto a desigualdad presupuestaria en la

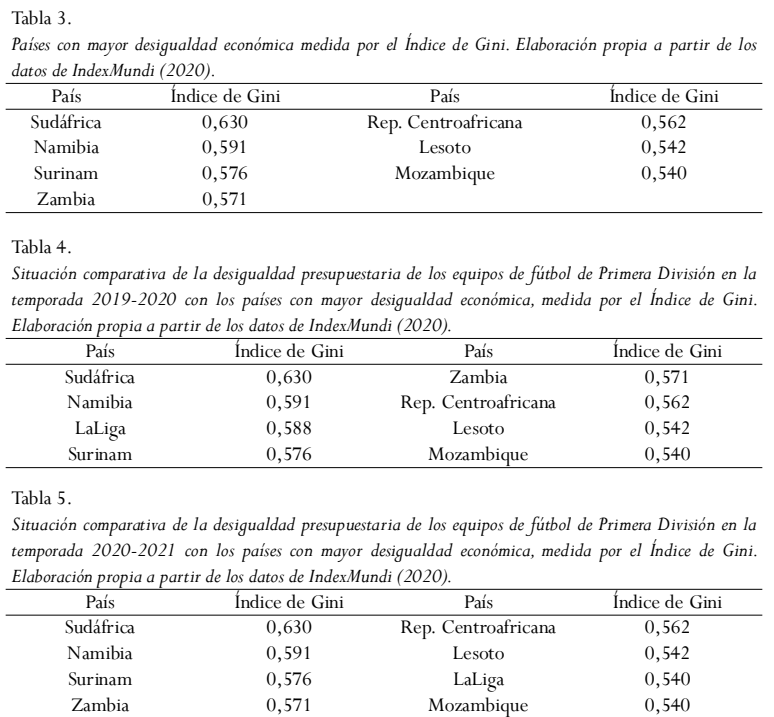


temporada 2020-2021.

Ahora procedemos a estudiar si el orden ha sufrido alguna diferencia significativa. De ser así, la minoración en el Índice de Gini habría sido estadísticamente significativa. Emplearemos el test de correlación por rangos de Spearman (Ruiz-Maya y Martín, 1995). Se trata de un contraste no paramétrico que parte de la hipótesis nula de ausencia de asociación entre los órdenes. Como el número de observaciones es pequeño, se utilizará la tabulación de Olds (Siegel, 1975).

De la aplicación ahora del test de correlación por rangos de Spearman para los órdenes de los países obtenidos en las tablas 4 y 5 , se obtiene un valor del coeficiente de 0,708 , con lo que estas variables estarían asociadas para un valor $p<0,05$ y la disminución del valor del Índice de Gini en LaLiga no sería significativa pero, sin embargo, sí que parece una disminución importante.

Si consideramos que el valor del Índice de Gini en la temporada 2020-2021 hubiera sido de 0,539 en lugar de 0,540 , es decir, si no hubiera existido esa coincidencia con Mozambique, si se hubiera descendido una milésima más, entonces el valor del coeficiente de Spearman sería de 0,642 , con lo que se aceptaría la hipótesis nula para un valor $p<0,05$ y no habría asociación entre los órdenes, lo que conllevaría a una diferencia significativa en el descenso del valor del Índice de Gini. Por eso solo hablamos de que se trata de una disminución importante, aunque no podemos decir que estadísticamente significativa.

\section{Conclusiones}

La desigualdad presupuestaria entre los clubes de Primera División en LaLiga ha disminuido entre las temporadas 2019-2020 y 2020-2021 debido a la pandemia del COVID-19 y a las repercusiones económicas que ha tenido.

En cualquier caso, la desigualdad presupuestaria entre los clubes de Primera División es muy alta. Para tener una idea más precisa de qué significa una desigualdad que provoque un Índice de Gini de 0,588. En la temporada 2020-2021, como consecuencia del COVID19, el Índice de Gini que hemos calculado desciende a 0,540 .

Se trata de una disminución que puede considerarse muy importante dentro de la desigualdad presupuestaria, aunque no alcanza a ser estadísticamente significativa, en sentido estricto, tal como acabamos de comprobar.
Esta desigualdad económica tiene un reflejo en los resultados obtenidos por los equipos de fútbol en las competiciones. Quizás fuera el momento de buscar propuestas que permitan reorganizar el sistema y reconducir la situación para conseguir una liga menos desigual.

En cualquier caso, el análisis de la desigualdad presupuestaria en la temporada 2021-2022 dará una idea nuevas repercusiones que pueda haber tenido el COVID19 y sus posibles efectos, con lo que se podría continuar la investigación.

\section{Referencias}

Alarcón, E.I. \& Hall-López, J.A. (2021). Physical activity in university student athletes, prior and in confinement due to pandemic associated with COVID-19. Retos, 39, 572-575.

Arés, R. (2020). La Covid merma las cuentas de la clase alta de LaLIga. Consultado el día 1 de abril de 2021 en https://as.com/futbol/2020/09/12/primera/1599869397_233274.html

AS (2020). Palmarés LaLiga Santander. Consultado el día 22 de marzo de 2021 en https:/ /as.com/especiales/futbol/primera/comunes/palmares/

Baena-Morales, S., López-Morales, J. \& García-Taibo, O. (2021). La intervención docente en educación física durante el periodo de cuarentena por COVID19. Retos, 39, 388-395.

Boor, S., Hanson, C. \& Ross, C. (2018). Football money league. Deloitte, Manchester (UK). Consultado el día 23 de marzo de 2021 en https:// www2.deloitte.com/content/dam/Deloitte/uk/ Documents/sports-business-group/deloitte-uk-sbgdfml2018.pdf

Burillo, P., Pérez-González, B. \& Salinero, J.J. (2012). ¿Liga bipolar? El balance competitivo de las principales ligas europeas de fútbol. En Calabuig, F., Molinos, M. \& Sala, R. (Eds.). Economía del deporte en tiempos de crisis, Proceedings del III Congreso Iberoamericano de Economía del Deporte, 7 y 8 de junio de 2012. Valencia (España).

Ceriani, L. \& Verme, P. (2014). Individual diversity and the Gini decomposition. Policy ResearchWorking Paper 6763. The World Bank. Washington DC (USA).

Coll, F. (2020). El impacto del COVID en el fútbol profesional español. Economipedia. Consultado el día 15 de julio de 2021 en https:/ / economipedia.com/ actual/el-impacto-del-covid-en-el-futbol-profesional-espanol.html 
Coll, S, \& Guijarro, M. (1998). Estadística aplicada a la historia y a las ciencias sociales. Ediciones Pirámide, Madrid (España).

Fuentes, R. (2020). Las fechas de la nueva liga: arranca el 11 de junio y acaba el 19 de julio. Diario SPORT. Consultado el día 15 de julio de 2021 en https:// www.sport.es/es/noticias/laliga/las-fechas-nuevaliga-arranca-junio-acaba-julio-7976645

García del Barrio, P. \& Pujol, F. (2008). El papel del fútbol en la sociedad actual. Fútbol: ocio y negocio. Revista Empresa y Humanismo, Vol. XI, I/08, pp. 89. 108.

Gay de Liébana, J.M. (2012). $5^{\circ}$ Informe anual sobre la situación económica del fútbol español y europeo. Consultado el día 22 de marzo de 2021 en https:// www.apmae.net/uploads / 6/9/0/7/6907416/ 5_informe_anual_situacion_economica_futbol_espanoleuropeo_2012.pdf

Herrera, C. (2016). Liga española busca un club que termine con el duopolio. El Economista, 16 de agosto de 2016. Consultado el día 22 de marzo de 2021 en https: / /www.eleconomista.com.mx/deportes/ Liga-espanola-busca-un-club-que-termine-con-elduopolio-20160818-0106.html

Hortigüela-Alcalá, D., Hernando-Garijo, A. \& PérezPueyo, A. (2021). Physical Education in the COVID19 context. A tale from teachers of different educational stages. Retos, 41, 764-774.

Huertos, M. \& Almorza, D. (2021). Análisis de la desigualdad en la Primera División de la liga española de fútbol. Training Fútbol 302, 1-4.

IndexMundi (2020). Recuperado el día 1 de abril de 2021 de https: / / www.indexmundi.com/es/datos/ indicadores/SI.POV.GINI/rankings

Mon, C. \& Rodríguez, P. (2016). El balance competiti- vo en la primera división de la liga de fútbol en España. Journal of Sports Economics \& Management, 6 (1), 18-34.

Montero, J.M. (2007). Estadística descriptiva. Thomson Editores, Madrid (España).

Mourao, P.R. \& Teixeira, J.S. (2015). Gini playing soccer. Applied Economics, Vol. 47, num. 49, pp. 5229-5246.

PCW (PricewaterhouseCoopers) (2018). Impacto económico, fiscal y social del fútbol porfesional en España, diciembre 2018. Consultado el día 22 de marzo de 2021 en https: / / files.laliga.es / 201902/28181426impacto-econ-mico- fiscal-y-social-del-f - tbol-pr.pdf

Pérez-González, B., Pérez-Espés, C., Cazurro, V. \& Gálvez, P. (2016). Estudio comparativo del balance competitivo de cinco ligas europeas de fútbol en el periodo 2000-2015. Revista Intercontinental de Gestao Desportiva, 6 (1), pp. 73-84.

RFEF (2020). Comisión de seguimiento por COVID-19:Vol. Acta de comisión (Número 1) (12 de marzo de 2020). Consultado el día 15 de julio de 2021 en https:/ / www.rfef.es / sites / default / files / pdf / acta_comision_seguimiento_12-3-20.pdf

Ruiz-Maya, L. y Martín, F.J. (1995). Estadística II:Inferencia. Editorial AC. Madrid (España).

Segui-Urbaneja, J., Juliao, R.P., Nogueira, R.M., Dorado, V. \& Farías-Torbidoni, E.I. (2021). Impacto de la COVID-19 en la práctica de personas participantes en eventos deportivos de carrera a pie y ciclismo en España y Portugal. Retos, 39, 743-749.

Siegel, S. (1975). Estadística no paramétrica aplicada a las ciencias de la conducta. Editorial Trillas (Méxi$\mathrm{CO})$.

Upon, G. y Cook, I. (2014). Dictionary of Statistics. Oxford University Press, Oxford (UK).

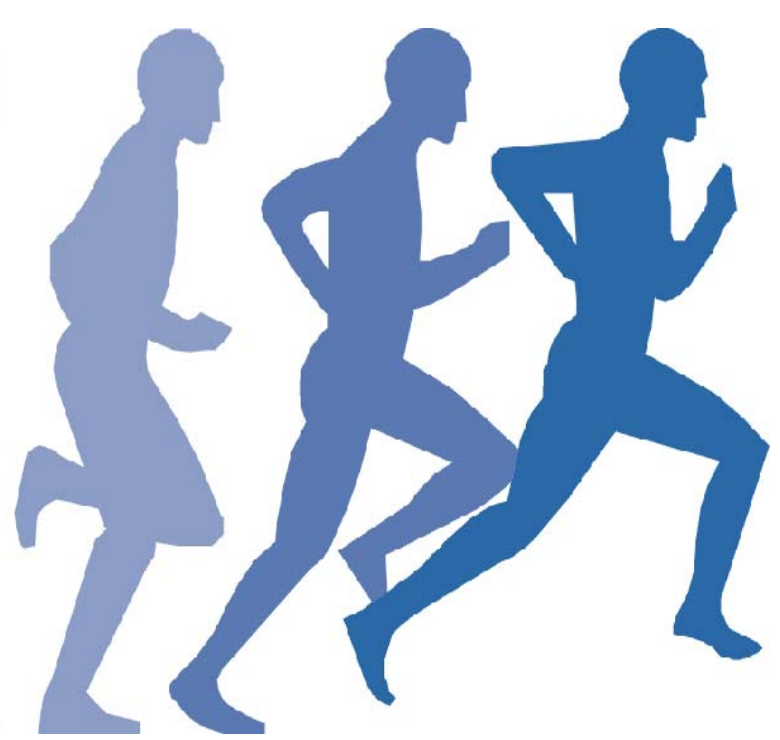

